\title{
Nachruf
}

Ansgar Büschges*

\section{Nachruf auf Prof. Dr. Dr. h. c. mult. Franz Huber}

https://doi.org/10.1515/nf-2017-0057

Am 27. April 2017 verstarb im Alter von 92 Jahren Prof. Dr. Dr. h. c. mult. Franz Huber, emeritierter Professor und ehemaliger Direktor am Max-Planck-Institut für Verhaltensphysiologie, Seewiesen.

Franz Huber wurde am 20. November 1925 in Nussdorf im Chiemgau geboren. Schon früh wurde bei dem Jugendlichen, der auf dem elterlichen Bauernhof aufwuchs, das Interesse für die Naturwissenschaften und besonders für das Verhalten von Tieren geweckt. Seine Schulzeit wurde wie für so viele junge Menschen seines Alters durch den Ausbruch des zweiten Weltkriegs und die Einberufung zum Arbeits- und Wehrdienst an der Ostfront unterbrochen. Nach seiner Rückkehr aus amerikanischer Kriegsgefangenschaft legte er im Jahr 1946 die „Kriegsteilnehmer“. Reifeprüfung am Gymnasium in Traunstein ab, woran sich zunächst eine Ausbildung als Zimmermann anschloss. 1947 begann Franz Huber das naturwissenschaftliche Studium der Biologie, Chemie, Physik und Geografie an der Ludwig-Maximilians-Universität und der Technischen Hochschule in München, welches er 1953 mit der Promotion im Fach Zoologie mit Bestnote abschloss.

Weite Teile seiner Studienzeit wurde Franz Huber als Stipendiat der Studienstiftung des Deutschen Volkes gefördert, eine Chance, die sicherlich auch die Basis seiner beruflichen Laufbahn, aber vor allem die von ihm stets gelebte Förderung von jungen Menschen in der Wissenschaft legte. Nach der Promotion ging er im Jahr 1954 an die Universität Tübingen, wo er im Jahr 1960 habilitierte. Franz Huber erhielt im Anschluss eine Reihe von Angeboten auf Professorenpositionen in den USA, z. B. von der University of Michigan und der University of California. Schlussendlich folgte er im Jahr 1963 dem Ruf auf das Ordinariat für Zoologie und Tierphysiologie an das Zoologische Institut der Universität zu Köln, das er bis 1973 innehatte. Dann folgte er dem Ruf an das Max-Planck-Institut für Verhaltensphysiologie in Seewiesen, dem er als einer der Direktoren vorstand. Bis zu seiner Emeritierung im Jahr 1993 leitete er am MPI Seewiesen die Abteilung Neuroethologie.

*Korrespondenzautor: Ansgar Büschges, Köln, Deutschland, E-Mail: ansgar.bueschges@uni-koeln.de
Seine wissenschaftliche Vitae wurde immer begleitet von einer enormen Begeisterung für wissenschaftliche Kooperationen im In- und Ausland, verbunden mit mehrfachen Aufenthalten in den jeweiligen Gastlaboren, z. B. an der Universität Zürich, der University of Uganda, der University of California at Los Angeles, am CalTech/ USA und an der University of Michigan/USA. Es waren vor allem seine vielfältigen Kooperationspartner in den USA und seine freundschaftlichen wissenschaftlichen Kontakte weltweit, mit denen Franz Huber wesentlich dazu beitrug, die europäische Tradition analytischer Verhaltensforschung sinnesphysiologischer Prägung in die (ganz anders strukturierte und stark wirbeltierzentrierte) Forschungslandschaft Nordamerikas zu tragen und dieses in Deutschland stark besetzte Feld international zu öffnen.

Der wissenschaftliche Lebensweg von Franz Huber ist gerade aus heutiger Sicht besonders bemerkenswert, weil er entlang seiner gesamten wissenschaftlichen Schaffensperiode „seinem“ Thema, welches er in seiner Doktorarbeit als für sich hochspannend identifiziert hatte, sehr erfolgreich treu bleiben konnte. Seine Doktorarbeit befasste sich mit den neuronalen Grundlagen der innerartlichen akustischen Kommunikation bei Grillen. Grillen gehören zu einer Insektengruppe, bei der Männchen mit dem Ziel der Partnerwahl spezifische Lautmuster mittels ihrer Flügel erzeugen, welche dann vorbehaltlich entsprechender Qualität zur Anlockung von weiblichen Artgenossen und zur anschließenden Paarung führen können. So konnte er durch Läsionsexperimente im Gehirn von Grillen unter anderem die Bedeutung bestimmter Areale für die Ausführung des Werbegesangs als notwendig nachweisen.

In seiner Postdoktorandenzeit (Universität Tübingen) eignete er sich neue elektrophysiologische Methoden an und verfeinerte so den Zugang zu den von ihm identifizierten Gehirnarealen der Grille und konnte lokalisierte Stimulation spezifischer Gehirnregionen durchführen (Gastaufenthalt an der Universität Zürich).

Wesentliche Erkenntnisse - nämlich zur Aufklärung der neuronalen Netzwerke, die für die Erzeugung innerartlicher Kommunikation bei Insekten verantwortlich sind (d.h. bei Männchen zur Erzeugung des Werbegesangs und bei Weibchen zur Gesangserkennung), prägten die Zeit seines wissenschaftlichen Schaffens als Ordinarius 
an der Universität zu Köln und dann als Direktor am MPI für Verhaltensphysiologie.

Aus eben jenen Untersuchungen wurde deutlich, dass die für die Gesänge der Männchen zu erzeugende rhythmische motorische Aktivität nicht von Netzwerken im Gehirn, sondern von spezialisierten Netzwerken im thorakalen Bauchmark erzeugt werden und dass neuronale Zentren im Gehirn die Kommandofunktion haben.

Franz Huber machte dieses Thema in den 1950er Jahren $\mathrm{zu}$ einem zentralen Schwerpunkt seiner Forschungsarbeit, zu einer Zeit, in der man kaum ahnen konnte, wie wichtig es in der aktuellen Forschung geworden ist: Derzeit erlebt die Entschlüsselung der Prinzipien der sog. „absteigenden Kontrolle“ im Nervensystem für die Erzeugung der vielfältigen Verhaltensweisen von Tieren (d. h. die Aufklärung der neuronalen Mechanismen, mittels derer übergeordnete Zentren im Gehirn prämotorische Netzwerke in Bauch- oder Rückenmark ansteuern und kontrollieren) eine besonders erfolgreiche Entwicklung. Gerade heute, wo es für einzelne Spezies, z. B. für die Fruchtfliege, möglich ist, durch neurogenetische Techniken eine systematische Identifikation der absteigenden Bahnen vom Gehirn inklusive ihrer funktionellen Analyse vorzunehmen, erlangen die von Franz Huber gelegten Erkenntnisse zu dieser Frage hohe Aktualität. Für das Nervensystem der Weibchen hingegen konnten Franz Huber und seine Mitarbeiter schon früh zeigen, dass spezifische Filtermechanismen entlang der neuronalen Hörbahn eine wesentliche Rolle für die Verarbeitung der innerartlichen Spezifität des wahrgenommenen Werbegesangs spielen.

Franz Huber veröffentlichte mehr als 120 Originalarbeiten und Übersichtsartikel. Bei drei Büchern firmierte er als Herausgeber.

In der Forschung folgte Franz Huber seiner Überzeugung, dass es unbedingt notwendig sei, neurophysiologische Analysen in den jeweils spezifischen Verhaltenskontext einzubetten; dabei habe der Experimentator Fragen nachzugehen, die ihm die Tiere selbst und nicht vorgefasste Konzepte stellten. Mit dieser Maxime trug Franz Huber wesentlich dazu bei, das Feld einer methodisch und konzeptionell breit aufgestellten, integrativen Verhaltensphysiologie zu präzisieren. Dies wird z. B. auch in seiner Rolle als Mitorganisator des Schwerpunktprogramms „Neuronale Mechanismen des Verhaltens“ der Deutschen Forschungsgemeinschaft deutlich.

Franz Huber hat mit wissenschaftlichem Weitblick und nie erlahmender Gestaltungskraft die Neuro- und Verhaltensbiologie in der zweiten Hälfte des letzten Jahrhunderts national und international in besonderer Weise geprägt. Dazu gehörte auch sein professioneller Einsatz für wissenschaftliche Fachzeitschriften, Gremien und Gesell-

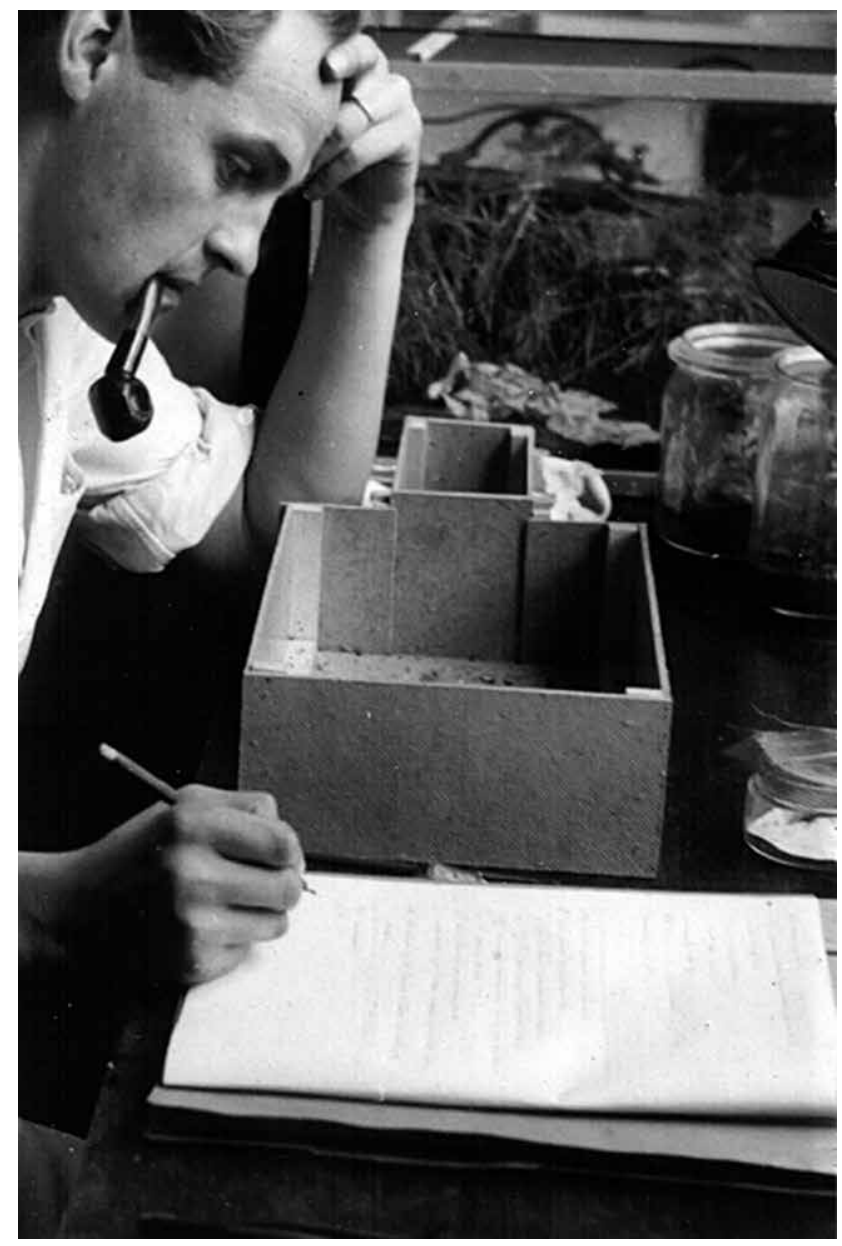

Abb. 1: Franz Huber bei der Verhaltensbeobachtung an Grillen im Jahr 1952

schaften: Franz Huber war Mitglied des Advisory Boards diverser Fachzeitschriften, z. B. Journal Comparative Physiology, Gutachter für die Deutsche Forschungsgemeinschaft, Beauftragter für die Max-Planck-Gesellschaft in diversen Gremien, Mitglied in verschiedenen Gremien der Deutschen Zoologischen Gesellschaft, welcher er schlussendlich 1993-1994 als Präsident vorstand. Die Deutsche Zoologische Gesellschaft würdigte seine Verdienste nicht nur mit der Karl-Ritter-von-Frisch-Medaille, sondern späterhin noch mit seiner Ehrenmitgliedschaft. Franz Huber galten diese beiden Auszeichnungen immer als besonders bedeutsam. Die Universitäten Köln, Toulouse, Odense und Zürich würdigten sein Wirken mit Ehrendoktoraten. Franz Huber war Mitglied der American Academy of Arts and Sciences, der American Philosophical Society, der Deutsche Akademie der Wissenschaften Leopoldina, sowie der Akademien der Bundesländer Bayern, Nordrhein-Westfalen und Rheinland-Pfalz. 2014 wurde er zum Fellow der International Society for Neuroethology ernannt. 


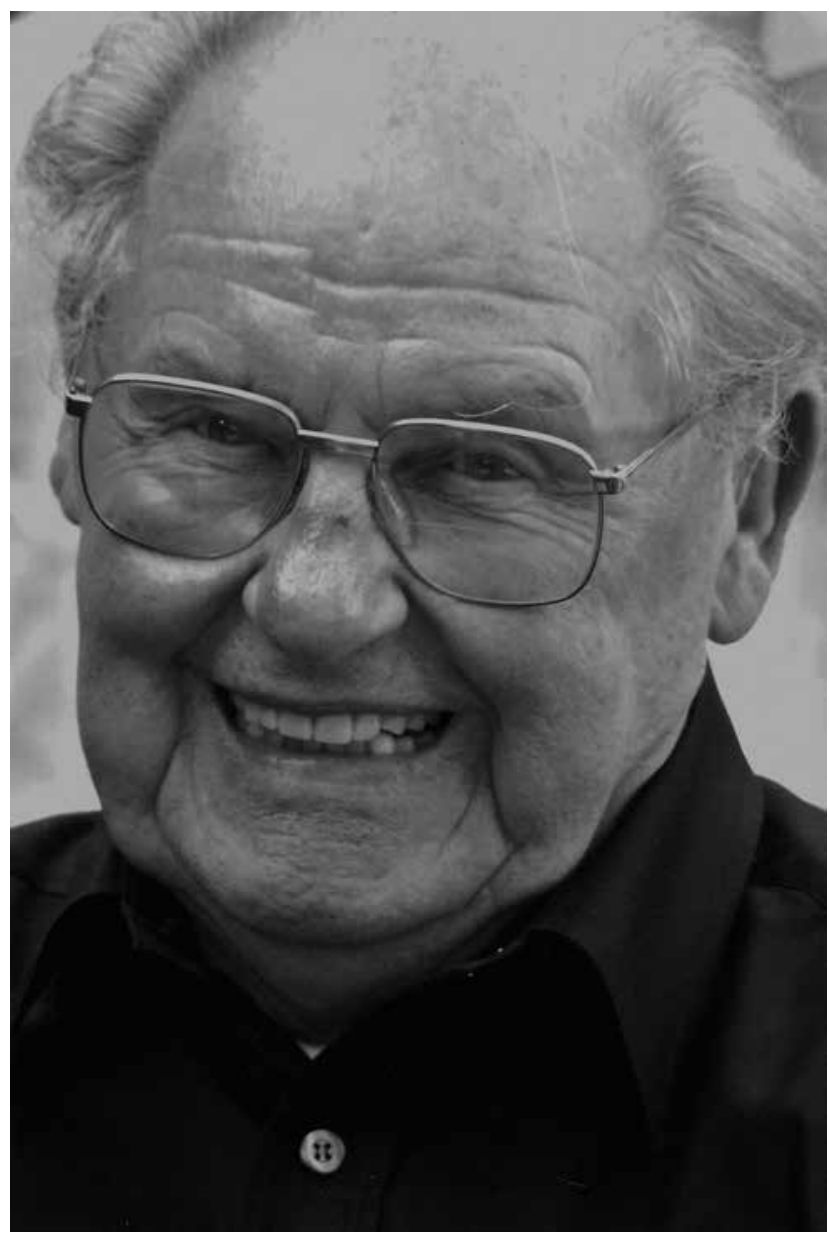

Abb. 2: Prof. Dr. Dr. h. c. mult. Franz Huber

Es ist Franz Huber selbst, der bei der Sicht auf seine wissenschaftlichen Verdienste immer wieder darauf verwies, dass diese ohne seine begabten MitarbeiterInnen und besonders ohne seine Ehegattin Lore nicht denkbar gewesen wären. Generationstypisch hatte Lore Schneider nach eigenem Studium und Promotion mit Auszeichnung an der Ludwigs-Maximilians-Universität in München ihrem Partner und der Familiengründung zuliebe auf eine weitere wissenschaftliche Laufbahn verzichtet. Besonders deutlich wird die Bedeutung von Lore Huber für ihren Ehemann aus den vielen persönlichen Erinnerungen Franz Hubers zu seinem Lebensweg, welche er im Jahr 2015 autobiografisch zugänglich machte (Das war mein Leben; Erinnerungen von Prof. Dr. Franz Huber).

Mit dem Tod von Franz Huber verliert die deutsche Neurowissenschaft einen ihrer renommiertesten Neuroethologen. Wir trauern um einen überaus geschätzten Kollegen, der einerseits ein wissenschaftlich erfolgreicher und aktiver Freund, Diskutant und Kollege, andererseits ein Vorbild für das professionelle Rollenverständnis eines erfahrenen Wissenschaftler war, weil er das lebte, was junge KollegInnen für die Arbeit in der Wissenschaft begeistert!

Generationen von deutschen und internationalen ZoologInnen und NeurowissenschaftlerInnen, denen es vergönnt war, mit Franz Huber zusammenzuarbeiten, mit ihm zu diskutieren oder seine Versuchsansätze und Arbeiten zu studieren, werden Franz Huber als Mensch und als einen der Väter der Neuroethologie in Erinnerung behalten.

Ansgar Büschges

Köln, Dezember 2017

Danksagung: Kollegen Rüdiger Wehner, auf dessen Nachruf (erschienen in Zoologie 2017, 93-99) meine Ausführungen in vielem fußen, möchte ich für die freundliche Durchsicht des Manuskripts herzlich danken. 
